\title{
Zone Axis STEM Defect Imaging Based on Electron Kossel Patterns
}

\author{
M.L. Bowers ${ }^{1}$, P.J. Phillips ${ }^{2}$, J. Kwon ${ }^{3}$, M.C. Brandes ${ }^{1}$, M.J. Mills ${ }^{1}$, and M. De Graef ${ }^{3}$ \\ 1. Department of Materials Science and Engineering, The Ohio State University, Columbus OH 43201 \\ 2. Department of Physics, University of Illinois at Chicago, Chicago IL 60607 \\ 3. Department of Materials Science and Engineering, Carnegie Mellon University, Pittsburgh PA 15213
}

Recently, scanning transmission electron microscopy (STEM) has been shown to provide numerous benefits to the practice of diffraction contrast imaging of crystalline defects [1,2]. Among others, a prominent benefit was the ability to perform defect imaging with the crystal aligned along a zone axis orientation, i.e., with the beam direction, $\vec{B}$, parallel to a principal zone. It was noted that the zone axis image quality, and hence defect contrast, are strongly dependent on the particular zone chosen. In this contribution, we propose that the defect image contrast quality from a given zone is directly related to the electron Kossel pattern (EKP), which is a function of crystal structure, orientation, and thickness, as well as the accelerating voltage.

The theory of EKP has been developed in conjunction with simulations of electron channeling contrast images (ECCI) of dislocations [3]. In essence, the EKP intensity is proportional to the number of incident beam electrons that "survive" through a given thickness, without being previously inelastically scattered. Hence, surviving electrons are then able to undergo elastic scattering events and give rise to defect contrast. EKP simulations were performed on multiple crystal systems for a variety of orientations. In addition, experimental STEM images were acquired on an FEI TF20 in crystals which contained various defects and were aligned along a zone axis orientation.

In Fig. 1, the central regions of electron Kossel patterns are presented for fcc-Ni at $200 \mathrm{kV}$, for a thickness of $100 \mathrm{~nm}$. Superimposed over each pattern are circles that represent the range of incident beam directions for three different convergence angles: 5.9, 8.2, and $12.2 \mathrm{mrad}$. For each convergence angle, the average intensity inside the circle was then calculated and normalized by the maximum intensity. The decimal value of this ratio for a $5.9 \mathrm{mrad}$ beam is indicated in the lower left corner of each pattern. Thus, a ratio of 1.00 represents the highest "transmittance" for a given convergence angle. Fig. 2 displays two example images acquired from a Ni-based superalloy oriented in zones that are considered to be favorable based on the EKPs. For these zones, not only is there high contrast between the matrix (Ni-based) and precipitates $\left(\mathrm{Ni}_{3} \mathrm{Al}\right.$-based), but there also exists desirable contrast between the defects and the microstructure. It is important to note that, habitually, we observe poor defect contrast quality when $\vec{B}=\langle 111\rangle$, which is consistent with its low EKP average intensity value.

Based on ample experimental evidence, larger normalized EKP intensity ratios correlate well with high quality zone axis STEM defect images. Thus, it is proposed that one can choose to perform defect imaging in certain zone axis orientations by using the calculated EKPs as a rubric for selection. In addition to the fcc case presented here, we will discuss the extension of this analysis (both EKP simulation and experimental imaging) to multiple other crystal systems, such as bec and hep.

References

[1] P.J. Phillips, M.J. Mills, and M. De Graef, Philosophical Magazine 91 (2011) 2081-2101. 
[2] P.J. Phillips, M.C. Brandes, M.J. Mills, and M. De Graef, Ultramicroscopy 111 (2011) 1483-1487.

[3] M. De Graef, Microscopy and Microanalysis, 18 (2012) 682-683.

[4] Portions of this work were funded under NSF Grant \#DMR-0907561.

[5] MDG was supported by the Air Force Office of Scientific Research, MURI contract \#FA9550-12-10458



Figure 1: Central regions of EKPs for fcc-Ni at $100 \mathrm{~nm}$ and $200 \mathrm{kV}$. The circles represent the range of incident beam orientations for 5.9, 8.2, and $12.2 \mathrm{mrad}$ convergence angles. Indicated in the lower left corner is the ratio of the average intensity inside the $5.9 \mathrm{mrad}$ circle, normalized by the maximum intensity value.

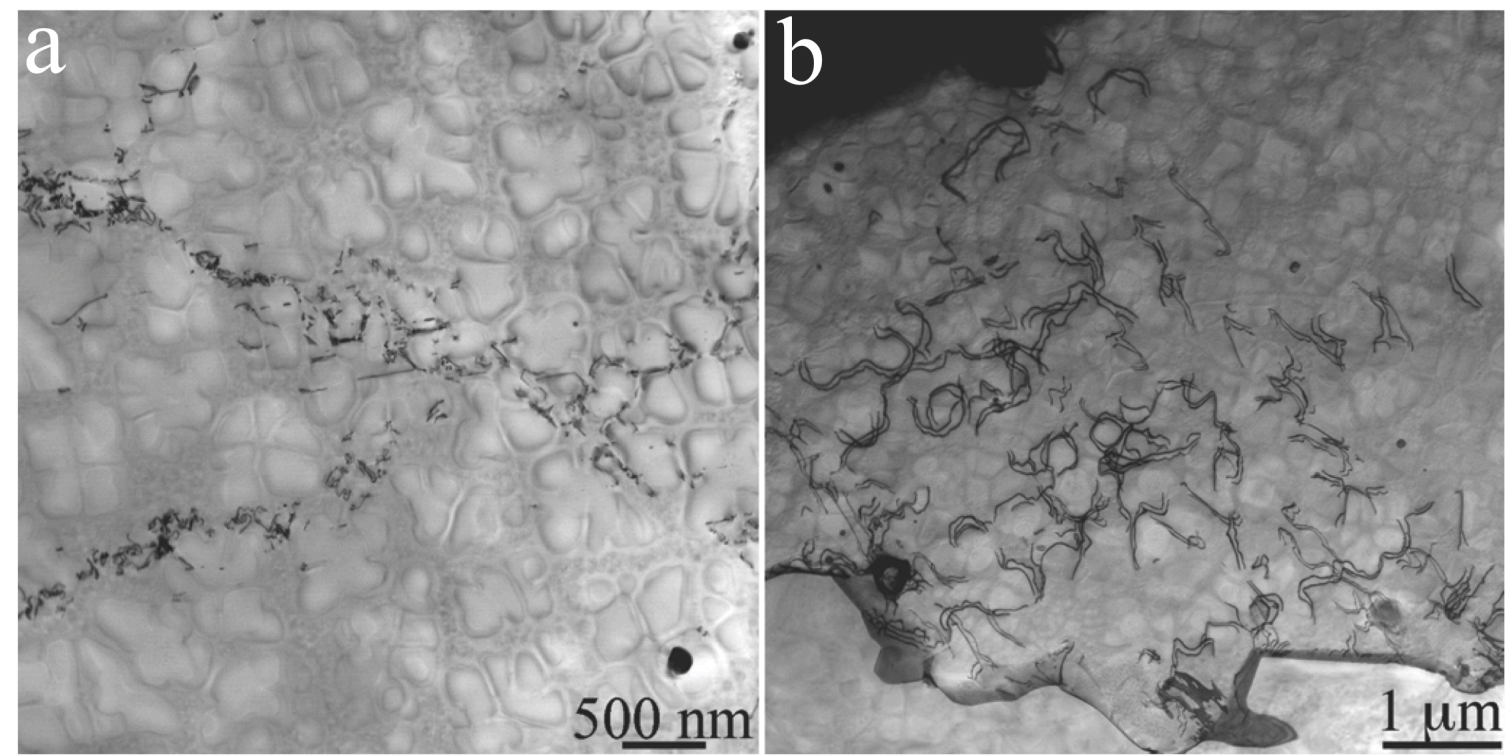

Figure 2: Zone axis STEM images of defected regions of a Ni-based superalloy acquired $\vec{B}=[001]$ (a) and $\vec{B}=[112] .(\mathrm{b})$ 